\title{
Role of right ventricular endomyocardial biopsy in infants and children with suspected or possible myocarditis
}

\author{
Steven A Webber, Gerard J Boyle, Ronald Jaffe, Ruth M Pickering, Lee B Beerman, \\ F Jay Fricker
}

\begin{abstract}
Objectives-To assess the diagnostic yield, sampling errors, risks, and therapeutic implications of right ventricular endomyocardial biopsy in children with suspected or possible myocarditis.

Design-Retrospective study.

Setting-Tertiary referral centre for paediatric cardiology, cardiac surgery, heart transplantation, and mechanical circula-
\end{abstract} tory support.

Patients and methods-Review of clinical and histological findings among 63 consecutive children with possible myocarditis undergoing right ventricular endomyocardial biopsy. Review of cardiac histology at subsequent necropsy or after explantation at time of transplantation. Results-From January 1980 to December 1992, 76 biopsies were performed in 63 children ( 2 weeks to 18 years of age). In 41 cases, the biopsy was performed for evaluation of dilated cardiomyopathy. The median interval from onset of symptoms was one month. Eight children (20\%; all with a history of less than six weeks duration) had biopsy proved myocarditis. Five of the eight children made a full recovery, including four who presented in cardiogenic shock. By contrast, only three of 33 children without evidence of myocarditis showed recovery of ventricular function. The whole heart was available for histological examination in 23 patients. Myocarditis was confirmed in one patient, and no evidence of myocarditis was found in the remaining 22 (all with negative biopsies). One procedure related death occurred in a 2 week old infant with dilated cardiomyopathy. In 22 cases, biopsy was performed for the evaluation of arrhythmia. Only one biopsy showed myocarditis.

Conclusions-The diagnostic yield of a biopsy is low in children with arrhythmias. In children presenting with profound ventricular dysfunction, a diagnosis of acute myocarditis may avoid premature consideration of transplantation as this group has an important potential for full recovery. In less critically ill patients and in those with a longer duration of symptoms the justification for biopsy is not as clear and the procedure is not without risk.

(Br Heart f 1994;72:360-363)
Myocarditis remains a histological diagnosis. The diagnostic yield and safety of endomyocardial biopsy in adults with cardiomyopathy has been well defined in large patient groups. ${ }^{12}$ Experience with endomyocardial biopsy in the assessment of myocarditis in children is far less extensive. ${ }^{3-7}$ In addition, sampling error has rarely been assessed in the paediatric age group as few hearts have subsequently been available for histological examination. We have therefore reviewed more than a decade of experience of right ventricular endomyocardial-biopsy in children with possible myocarditis, with the aims of assessing procedural safety, diagnostic yield, sampling errors, and therapeutic implications.

\section{PATIENTS AND METHODS}

We retrospectively reviewed data on all patients undergoing an endomyocardial biopsy from January 1980 to December 1992 at our institution. Patients were included in this review if a principal aim of the biopsy was to establish or refute a diagnosis of myocarditis. Thus patients with hypertrophic or restrictive cardiomyopathies were excluded, as were patients undergoing biopsy for the evaluation of rejection after heart transplantation.

Patients fell into two distinct clinical groups: those with unexplained ventricular dysfunction and those with arrhythmias (usually ventricular), but a structurally normal heart. All but two of the patients with ventricular dysfunction presented with cardiac failure. One child presented with fever and a newborn infant was initially considered to have persistent pulmonary hypertension. Ventricular function was moderately or severely impaired at presentation (median shortening fraction $12 \%$; range $0-22 \%$ ). Four patients presented in cardiogenic shock and one of these children sustained cardiac arrest shortly after admission to hospital. Biopsies were performed using a femoral or right internal jugular venous approach and four to seven biopsy specimens were obtained for each patient. All medical records were reviewed. Histology was reviewed by one pathologist (RJ) with a special interest in cardiac histopathology. This review was performed without knowledge of the initial pathology report or clinical course. Myocarditis was diagnosed according to the Dallas criteria - that is, both inflammatory infiltrate and adjacent myocyte damage were required for the diagnosis of definite myocarditis. ${ }^{8}$ Whenever available, findings at endomyocardial biopsy were 
contrasted with those obtained at necropsy or after explanation at the time of transplantation.

\section{Results}

Over the 13 year period, 76 right ventricular endomyocardial biopsies were performed in 63 children with possible myocarditis.

\section{CARDIOMYOPATHY GROUP}

There were 41 children with cardiomyopathy (24 boys), with a mean age of 7 years (range 2 weeks to 18 years, median $7 \cdot 2$ years). Time from onset of symptoms to biopsy varied widely ( 1 day to 150 months), but was short in most patients (median one month; interquartile range 0.4-3.3 months). Eight $(20 \%)$ of these children were diagnosed histologically as having definite myocarditis. If biopsy had been limited to those with a history of less than six weeks (24 patients), all eight patients with myocarditis would have been identified (diagnostic yield 33\%). The shortening fraction at presentation among the eight children with myocarditis ranged from zero (undetectable ventricular shortening) to $17 \%$ (median $9 \%$ ). All four patients presenting in cardiogenic shock had histological evidence of active myocarditis.

One child showed a spontaneous and full recovery of ventricular function associated with evidence of healed myocarditis on early follow up biopsy. A second child showed continued deterioration in ventricular function with evidence of ongoing myocarditis on a second biopsy. He underwent successful orthotopic transplantation within a month of presentation, without previous immunosuppressive treatment. The remaining six children with myocarditis (including the four presenting in cardiogenic shock) received immunosuppressive treatment with corticosteroids alone or corticosteroids with cyclosporine. Four of the six had serial endomyocardial biopsies (total 10 follow up biopsies) during and after the end of immunosuppressive treatment. At the time of the last biopsy, two had evidence of healed myocarditis, one of resolving myocarditis and one of ongoing myocarditis (but with less pro-

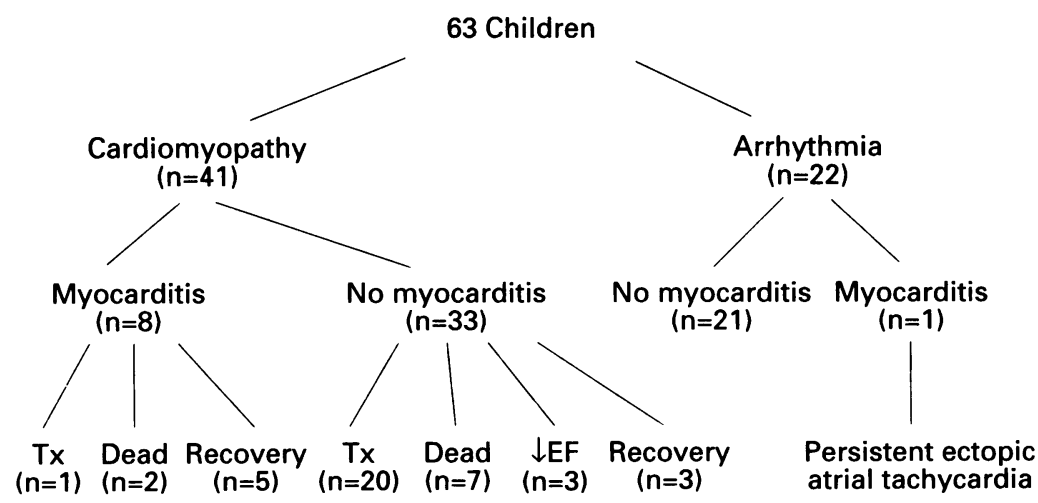

Patient details and outcome. $T x=$ transplantation; and $E F=$ ejection fraction. nounced lymphocytic infiltration). All four patients presenting in cardiogenic shock made a full recovery, including two who required mechanical circulatory support using extra corporeal membrane oxygenation. Two late sudden deaths occurred, two and 14 months after presentation, in patients with subacute presentation who received immunosuppressive treatment. Neither had recovered normal ventricular function during treatment with corticosteroids. Among the 33 patients with dilated cardiomyopathy without evidence of myocarditis, the prognosis was much worse. Twenty underwent orthotopic transplantation, seven died, and three have residual ventricular dysfunction (figure).

\section{ARRHYTHMIA GROUP}

Twenty two children underwent a biopsy for the evaluation of arrhythmia (ventricular tachycardia, 18; ectopic atrial tachycardia, two; atrial flutter with ventricular ectopy, one; and acute onset complete heart block, one). Their mean age was 10.5 years (range $0.7-18$ years; median $12 \cdot 3$ ) and the time from the onset of symptoms to biopsy was 0-72 months (median 0.7 months, interquartile range $0 \cdot 25-2$ months). Minor histological abnormalities were common, occurring in 17 of the 22 patients. These abnormalities included myocyte or myocyte nuclear hypertrophy (10 patients), increased interstitial fibrosis (five patients), and interstitial oedema (four patients). One child with ventricular tachycardia had unusually small myocytes with a paucity of cytoplasm compared with the nuclear content. Only one child (with acute onset atrial ectopic tachycardia) had active myocarditis. Follow up biopsy after 10 weeks of corticosteroid treatment showed ongoing (though less severe) myocarditis, with the patient in persistent ectopic tachycardia.

\section{HISTOLOGY AND OUTCOME}

The whole heart was available for examination in 24 of the 63 patients (38\%). This was obtained at the time of transplantation in 20 patients and at necropsy in four. Twenty three of these patients presented with dilated cardiomyopathy and one with ventricular tachycardia. The time between biopsy and examination of the whole heart ranged from one day to 2.6 years, but was generally fairly short (median 2.4 months, interquartile range $0 \cdot 4-6.0$ months). Only one of these patients had myocarditis diagnosed on endomyocardial biopsy and this was confirmed at the time of explantation of the heart six weeks later. Among the 23 remaining patients, none had histological features of myocarditis when the whole heart was subsequently examined. None of these patients had received immunosuppressive treatment between the time of biopsy and death or transplantation.

One 2 week old infant with dilated cardiomyopathy developed cardiac tamponade during the biopsy procedure and could not be resuscitated. There were no other complications. 


\section{Discussion}

Myocarditis is a rare disease and its treatment remains controversial. An endomyocardial biopsy is often performed in adult patients with suspected myocarditis to select those felt to be suitable for immunosuppressive treatment. We, like others, ${ }^{9}$ have also taken this approach in paediatric patients, although we recognise that no randomised trials of treatment have been performed in this age group. The recent Multicentre Treatment Trial for Myocarditis has shown no clear benefit from immunosuppressive treatment in adult patients with biopsy proved myocarditis (Mason J, unpublished data). This has led to further doubts about the role of routine endomyocardial biopsy in patients with suspected or possible myocarditis. ${ }^{10}$

Our observations confirm the rare nature of myocarditis in children. We identified only nine biopsy proved cases over a 13 year period in a large paediatric cardiology referral centre with active transplantation and mechanical circulatory support programmes. We have identified only five additional patients over the study period in whom myocarditis was diagnosed at necropsy or during examination of an explanted heart, but in whom endomyocardial biopsy had not been performed. Our diagnostic yield of biopsy in patients with cardiomyopathy was approximately $20 \%$, which is higher than that in two large adult studies (Mason J, unpublished data). ${ }^{1}$ This might represent a chance effect because of our relatively small sample size, an unusual patient group, or possibly a difference between paediatric and adult populations. Our data also show that limiting biopsy to patients with a short duration of symptoms will significantly increase this diagnostic yield. The diagnostic yield in our series increased to $33 \%$ when biopsy was performed in patients with a history of less than six weeks. No cases of myocarditis were identified when the history exceeded this period.

Our one procedural related death in a 2 week old infant confirms that biopsy is not without hazard, and this may be particularly true in the newborn period, when the right ventricle is relatively thin.

Previous studies in children have been unable to assess sampling error as few hearts have been subsequently examined at necropsy or explantation. Although there is an inevitable delay between the time of biopsy and whole heart examination, our data suggest that sampling error (that is, false negative biopsies) is probably rare in children, if adequate numbers of samples are obtained (usually four to six for each patient). We cannot rule out the possibility, however, of false negative biopsies with subsequent healing of myocarditis by the time of subsequent histological examination of the whole heart. False negative biopsies (that is, undiagnosed myocarditis) might explain the recovery of three of the 33 patients considered to have idiopathic dilated cardiomyopathy.

On whom should we perform a biopsy? We would concur with the editorial com- ments of Davies and Ward ${ }^{10}$ that a biopsy for the purpose of histological diagnosis is no longer routinely justified. If biopsies are to be performed in children, we believe they should be performed as early as possible in the course of the clinical illness and ideally should be restricted to the setting of controlled clinical trials of treatment. Our current data suggests, however, that such trials will be an enormous undertaking. If we assume, for example, that spontaneous recovery occurs in about $50 \%$ of patients with myocarditis, then the detection of a $70 \%$ recovery rate at the $5 \%$ level of significance in the treatment arm of a simple two arm trial would require 93 patients in each group to achieve $80 \%$ power. $^{11}$ If the biopsy is restricted to patients with a history less than six weeks, approximately 600 children with possible myocarditis would be required to define a population suitable for such a trial (assuming a diagnostic yield of approximately one third). Furthermore, this assumes that all patients subsequently enter and complete the study. In the adult Multicentre Treatment Trial for Myocarditis, biopsies were performed in over 2000 patients and although $209(10 \%)$ were initially considered to have myocarditis (by the local pathologist), only $111(6 \%)$ were subsequently randomised to receive immunosuppressive treatment or placebo. Many of these were later considered not to have myocarditis when an expert panel reviewed the histological findings. ${ }^{10}$ These observations raise doubts about the realistic possibility of a similar trial in children.

We have shown that a biopsy may sometimes have an important role in defining prognosis. This may be particularly relevant when a child is being considered as a candidate for urgent transplantation. Four of our patients (all of whom survived) presented in cardiogenic shock and two required mechanical circulatory support. If a histological diagnosis of myocarditis can be made in these critically ill patients, then time can be given for recovery to occur, before transplantation is considered. In less critically ill patients, the justification for routine biopsy appears less clear. Although the benefits of immunosuppressive treatment remain unproved, we continue to consider their use in occasional patients with persistent cardiac failure unresponsive to conventional treatment and in patients with poor systolic function who do not show spontaneous recovery. Because all immunosuppressive drugs have important side effects, we believe such empirical trials of treatment should only be instituted in patients with biopsy proved myocarditis.

Endomyocardial biopsy should continue to have a role as a research tool in increasing our understanding about the aetiology of myocarditis. Amplification of viral genome from biopsy specimens is already giving new insights into the spectrum of viruses causing myocarditis in childhood. ${ }^{12}$ As new antiviral drugs are developed, biopsy may more directly influence clinical decision making. 
1 Herskowitz A, Cambell S, Deckers J, et al. Demographic features and prevalence of idiopathic myocarditis in patients undergoing endomyocardial biopsy. $A m \boldsymbol{f}$ patients undergoing

2 Starling RC, Van Fossen DB, Hammer DF, Unverferth DV. Morbidity of endomyocardial biopsy in cardiomyopathy. Am F Cardiol 1991;68:133-6.

3 Lewis AB, Neustein HB, Takahashi M, Lurie PR Findings on endomyocardial biopsy in infants and children with dilated cardiomyopathy. Am $\mathcal{F}$ Cardiol 1985 55:143-5.

4 Nihill MR, Bricker JT, Kearney DK. Endomyocardial biopsy in infants and children. In: Boyle EF, Engle MA Gersony WM, Rashkind WJ, Talner NS, eds. Proceedings of 2 nd World Congress of Pediatric Cardiology. New York: Springer-Verlag, 1986:342-7.

5 Leatherbury L, Chandra RS, Shapiro SR, Perry LW Value of endomyocardial biopsy in infants, children and adolescents with dilated or hypertrophic cardiomyopathy and mon

6 Schmaltz AA, Jurgen A, Waldemar H, Maisch B. Endo- myocardial biopsy in infants and children: experience in 60 patients. Pediatr Cardiol 1990;11:15-21.

7 Wiles HB, Gillette PC, Harley RA, Upshur JK. Cardiomyopathy and myocarditis in children with ventricular ectopic rhythm. $\mathcal{F}$ Am Coll Cardiol 1992;20: tricular

8 Aretz HT, Billingham ME, Edwards WD, et al. Myocarditis: a histopathologic definition and classification. Am $\mathcal{F}$ Cardiovasc Pathol 1986;1:3-14.

9 Chan KY, Iwahara M, Benson LN, Wilson GJ, Freedom RM. Immunosuppressive therapy in the management of acute myocarditis in children: a clinical trial. $\Im \mathrm{Am}$ Coll Cardiol 1991;17:458-60.

10 Davies MJ, Ward DE. How can myocarditis be diagnosed and should it be treated? [editorial]. Br Heart $\mathcal{F} 1992$; 68:346-7.

11 Machin D, Campbell MJ. Statistical tables for the design of clinical trials. Oxford: Blackwell Scientific, 1987.

12 Martin A, Webber SA, Fricker FJ, et al. Acute myocarditis: diagnosis by PCR in children. Circulation 1994;90: 330-9. 